\title{
The Comorbidity Burden of Hidradenitis Suppurativa in the United States: A Claims Data Analysis
}

\author{
Alexandra B. Kimball · Murali Sundaram - Genevieve Gauthier • \\ Annie Guérin · Irina Pivneva · Rakesh Singh · Arijit Ganguli
}

Received: July 23, 2018 / Published online: October 10, 2018

(C) The Author(s) 2018

\section{ABSTRACT}

Introduction: Prior studies have reported that hidradenitis suppurativa (HS) is accompanied by a myriad of physical and mental conditions. However, given the small sample sizes and the limited number of pre-selected comorbidities,

Enhanced Digital Features To view enhanced digital features for this article go to https://doi.org/10.6084/ m9.figshare.7098395.

Electronic supplementary material The online version of this article (https://doi.org/10.1007/s13555018-0264-z) contains supplementary material, which is available to authorized users.

\section{A. B. Kimball ( $\square)$}

Harvard Medical Faculty Physicians at Beth Israel

Deaconess Medical Center, Boston, MA, USA

e-mail: clears@bidmc.harvard.edu

\section{Sundaram}

AbbVie Inc, North Chicago, IL, USA

G. Gauthier · A. Guérin · I. Pivneva

Analysis Group, Inc, Montreal, Canada

R. Singh · A. Ganguli

Global Health Economics and Outcomes Research,

AbbVie Inc, North Chicago, IL, USA

Present Address:

M. Sundaram

Janssen Scientific Affairs, Horsham, PA, USA

Present Address:

G. Gauthier

StatLog Inc., Montreal, Quebec, Canada these studies do not provide a complete picture of the comorbidity burden of HS in the USA. Moreover, the relationship between HS severity and comorbidity burden has yet to be characterized. Using a large US claims database, we estimated the comorbidity burden associated with HS, stratified by disease severity.

Methods: A retrospective matched cohort design was used. Patients with HS were classified into two severity cohorts (milder and more severe) using an empirical algorithm based on treatments received. The comorbidity burden was compared between each HS cohort and their matched HSfree cohort, and between patients with milder vs. those with more severe forms of HS.

Results: Several physical and mental comorbidities were found to be more prevalent in both cohorts of patients with milder and more severe forms of HS than in their matched HS-free cohorts. The comorbidity burden also increased greatly as the disease progressed to more severe forms.

Conclusions: The results of this study highlight the complexity of the comorbidity burden of HS patients and the need for a multidisciplinary approach to optimize the management of HS and its numerous associated comorbidities.

Funding: AbbVie, Inc.

Keywords: Comorbidity burden; Hidradenitis suppurativa; Severity 


\section{INTRODUCTION}

Hidradenitis suppurativa (HS) is a chronic, relapsing follicular occlusion disease primarily affecting intertriginous areas of the body, such as axillary and inguinal regions [1]. Its worldwide prevalence is estimated to vary between 0.05 and $4.10 \%$, based on the methodology used [2]. In the early stages of the disease, manifestations include isolated papules or nodules that spread and become deep-seated abscesses with tunneling tracts as the disease progresses [1, 3]. In the most advanced stages of the disease, interconnected sinus tracts form between abscesses and extensive scarring occurs $[1,3,4]$.

In addition to its typical clinical manifestations, HS is often accompanied by other follicular occlusion diseases, such as acne conglobata, dissecting cellulitis of the scalp, and pilonidal cyst [5-7]. Inflammatory conditions have also been reported to be frequent in HS patients, including arthritides, autoimmune conditions, metabolic syndrome, and inflammatory bowel disease [7-16]. Several case studies have also documented the co-occurrence of hypertension [16], asthma [17], deficiency anemias [17], kidney disease $[11,18]$, herpes simplex [15], and rare congenital disorders, such as ichthyosis congenital disorder, keratitis ichtyhosis-deafness [7], and Down syndrome [7, 19]. Besides physical comorbidities, HS has also been associated with mental comorbid conditions, such as depression and anxiety. Most of the somatic and psychiatric comorbidities in HS patients are linked to the increased expression of pro-inflammatory cytokines, such as tumor necrosis factor alpha, interleukin (IL)1, IL17, and IL32 [20-22]. Finally, although squamous cell carcinoma is relatively rare, it has been reported as one of the most severe complications of HS, with a mortality rate of approximately 50\% among HS patients. It has been hypothesized that the development of epithelial malignancies in the context of chronic HS may be due to an impaired local immunity [23-25].

Although a number of studies have reported that HS is accompanied by a multitude of physical and mental conditions, evidence reported in current literature has been largely based on case reports or series of hospitalized or specialty clinic populations and does not provide a complete picture of the comorbidity burden among the general HS patient population in the USA. Only a few comorbidities, such as obesity and diabetes, have been shown to be associated with disease severity [26]. However, no studies have described and compared the overall comorbidity burden associated with milder versus more severe forms of HS. Therefore, the objective of this study was to estimate the comorbidity burden associated with HS stratified by disease severity.

\section{METHODS}

\section{Data Source}

Patients were selected from the OptumHealth Care Solutions, Inc. database (January 1999-March 2014), an administrative claims database containing medical and pharmacy claims for approximately 14 million privately insured beneficiaries (employees, spouses, and dependents) from 60 self-insured large employers located across the USA. Data in this database are de-identified and comply with the patient confidentiality requirements of the Health Insurance Portability and Accountability Act.

This was a retrospective analysis of anonymized data; no institutional board review was required.

\section{Sample Selection and Study Design}

Patients with at least two diagnoses for HS (International Classification of Diseases, Ninth Revision, Clinical Modification [ICD-9-CM] code 705.83) recorded on different dates between January 1999 and March 2014 were selected. Each HS diagnosis date was identified as a potential index date. Selected patients were required to be at least 18 years of age as of the index date and continuously enrolled in a healthcare plan during the 18-month study period, which spanned 6 months before and 12 months after the index date. All HS patients meeting the selection criteria were included. For HS patients with more than one potential index date meeting the selection criteria, the study 
index date was randomly selected using an equal probability method.

As HS severity is not currently coded in claims data, it was proxied based on the presence of indicators of more severe forms of HS (described in Table 1). Among the selected HS patients,

Table 1 Indicators of more severe forms of hidradenitis suppurativa

\begin{tabular}{l} 
Indicators of more severe forms of $\mathbf{H S}^{\mathbf{a}}$ \\
\hline Inpatient/emergency room admission-with a diagnosis \\
of HS on the same day \\
Skin surgery-with a dermatological-related diagnosis \\
on the same day \\
Treatments-within 7 days of an HS diagnosis \\
Antibiotics (oral $\geq 90$ days) \\
Antimycobacterials (oral $\geq 90$ days) \\
Anti-infectives (oral $\geq 90$ days) \\
Corticosteroids (oral $\geq 90$ days) \\
Intralesional steroids ( $\geq 4$ per year) \\
Biologics \\
Dapsone \\
Metformin \\
Prostatic hypertrophy agents \\
Retinoids \\
Selected anti-androgens/hormonals \\
\hline
\end{tabular}

$H S$ Hidradenitis suppurativa

a Patients who experienced at least one of the disease severity indicators during the study period were classified as patients with more severe forms of HS (HS-severe). HS patients who did not experience any of the indicators of more severe forms of HS were classified as patients with milder forms of HS (HS-mild)

b Dermatological-related diagnoses included HS, pilonidal cyst, sebaceous cyst, other cellulitis and abscess, other inflammatory disorders of male genital organs, other abscess of vulva, and other acne

c Other selected treatments included azathioprine, cyclosporine, leflunomide, methotrexate, and mycophenolate patients who experienced at least one of the disease severity indicators during the study period were classified as patients with more severe forms of HS (HS-severe). HS patients who did not experience any of the indicators of more severe forms of HS were classified as patients with milder forms of HS (HS-mild).

A retrospective matched cohort design was used to match patients with milder and more severe forms of HS 1:1 to HS-free control patients based on available demographic characteristics, including the year of birth, sex, region of residence, and healthcare plan. To minimize selection bias, we randomly selected controls among all patients meeting the section selection criteria without any diagnosis for HS over the entire period covered by the database. For the patients in the HS-free cohorts, the index date was defined as the same calendar date as that of their matched HS cohort.

Outcomes during the study period were compared between (1) patients with milder forms of HS and their matched HS-free controls, (2) patients with more severe forms of HS and their matched HS-free controls, and (3) patients with milder and more severe forms of HS.

\section{Definition of Outcome and Statistical Analyses}

Patient comorbidities were identified based on medical claims recorded during the study period. Comorbidities reported in the literature to be frequently associated with HS and other physical and mental comorbidities, based on Elixhauser et al. and the Diagnostic and Statistical Manual of Mental Disorders, Fifth Edition (DSM-5) [27-29], were analyzed.

Patient characteristics and prevalence of comorbidities were summarized using means, medians, and standard deviations for continuous variables and counts and percentages for categorical variables, and also compared between HS patients and their matched HS-free controls using Wilcoxon signed-rank tests for continuous variables and McNemar tests for categorical variables. Comparisons between patients with milder forms of HS and patients with severe forms of HS were conducted using 
Wilcoxon rank-sum tests for continuous variables and Chi-squared tests for categorical variables. Bonferroni correction was applied to adjust for multiple comparisons. All analyses were conducted using SAS version 9.4 (SAS Institute Inc., Cary, NC, USA).

\section{RESULTS}

A total of 5357 patients with HS were matched to HS-free controls. Of these patients, 2292 had milder forms of HS and 3065 had more severe forms of HS (Fig. 1).
Overall, the majority of HS patients were female $(72.3 \%)$, with an average age of 42.2 years. Patients with milder and more severe forms of HS were not significantly different in terms of age and region of residence although there were some minor differences with respect to types of healthcare plan; for example, compared with patients with milder forms of HS, a higher proportion of those with more severe forms of HS were enrolled in point of service and a lower proportion were enrolled in a feefor-service types of plan (Table 2).

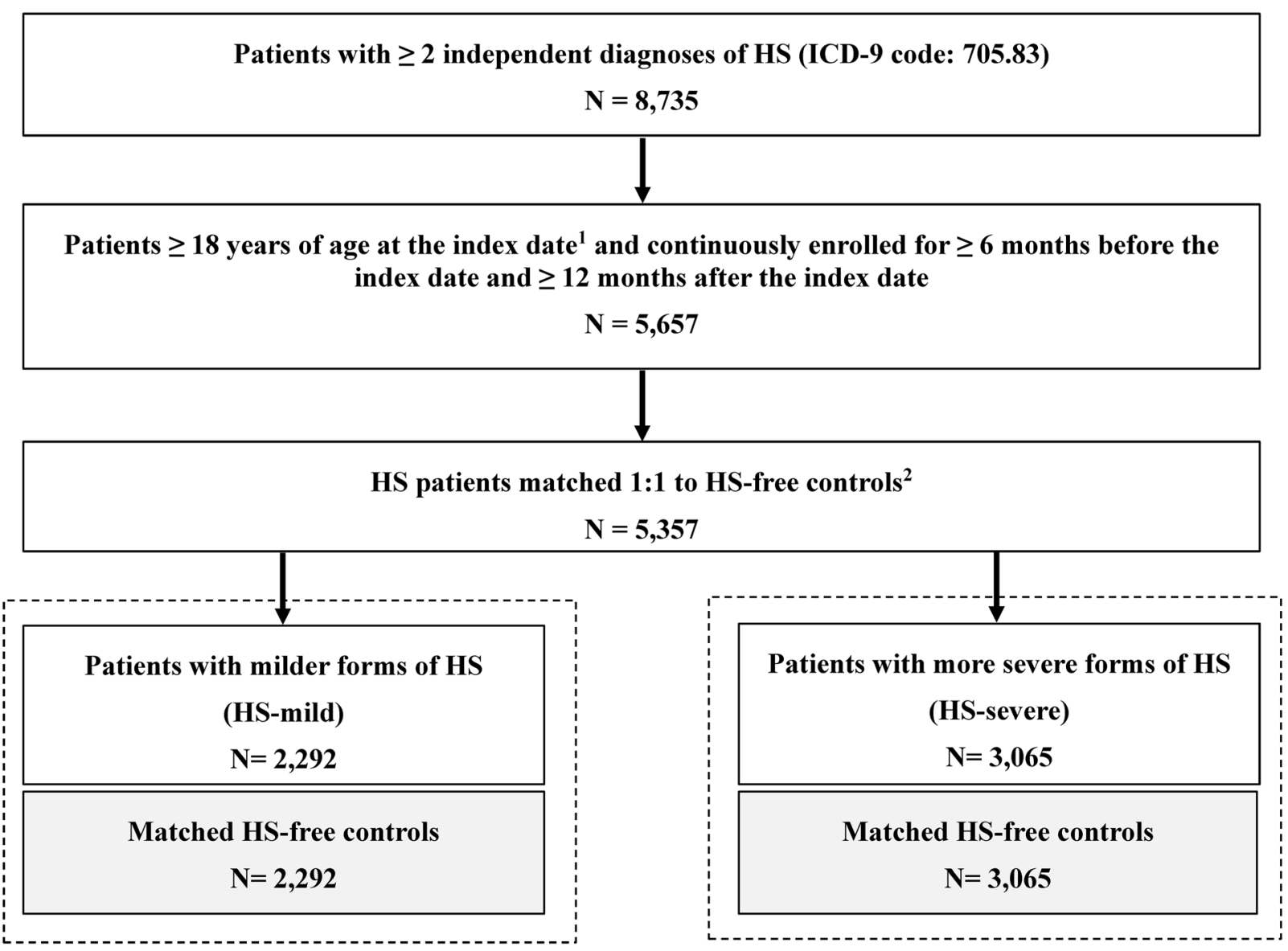

Fig. 1 Flow chart of sample selection. HS hidradenitis suppurativa, ICD-9 International Classification of Diseases, 9th Edition. ${ }^{1}$ Index date is the date of the HS diagnosis. ${ }^{2}$ Matched HS-free control patients were required to be $\geq 18$ years of age at the index date and continuously enrolled for $\geq 6$ months before the index date and for $\geq 12$ months after the index date 
Table 2 Patient characteristics at baseline

\begin{tabular}{|c|c|c|c|c|c|}
\hline \multirow{2}{*}{$\begin{array}{l}\text { Patient characteristics at } \\
\text { baseline }\end{array}$} & \multicolumn{2}{|c|}{ Matched cohorts } & \multicolumn{2}{|c|}{ Matched cohorts } & \multirow{2}{*}{$\begin{array}{l}p \text { value }^{\text {a }} \text { HS-mild vs. } \\
\text { HS-severe }\end{array}$} \\
\hline & $\begin{array}{l}\text { HS-mild } \\
(N=2292)\end{array}$ & $\begin{array}{l}\text { HS-free } \\
(N=2292)\end{array}$ & $\begin{array}{l}\text { HS-severe } \\
(N=3065)\end{array}$ & $\begin{array}{l}\text { HS-free } \\
(\boldsymbol{N}=3065)\end{array}$ & \\
\hline Age, mean (years) ${ }^{b}$ & $\begin{array}{l}42.19 \pm 15.24 \\
{[42]}\end{array}$ & $\begin{array}{l}42.19 \pm 15.24 \\
{[42]}\end{array}$ & $\begin{array}{l}42.19 \pm 14.16 \\
{[42]}\end{array}$ & $\begin{array}{l}42.19 \pm 14.16 \\
{[42]}\end{array}$ & 0.6520 \\
\hline $18-24$ & $367(16.0)$ & $367(16.0)$ & $409(13.3)$ & $409(13.3)$ & $0.0060^{*}$ \\
\hline $25-40$ & $754(32.9)$ & $754(32.9)$ & $1060(34.6)$ & $1060(34.6)$ & 0.1967 \\
\hline $41-64$ & $988(43.1)$ & $988(43.1)$ & $1426(46.5)$ & $1426(46.5)$ & $0.0128^{*}$ \\
\hline$\geq 65$ & $183(8.0)$ & $183(8.0)$ & $170(5.5)$ & $170(5.5)$ & $0.0004^{*}$ \\
\hline Female & $1697(74.0)$ & $1697(74.0)$ & $2176(71.0)$ & $2176(71.0)$ & $0.0137^{*}$ \\
\hline \multicolumn{6}{|l|}{ Region } \\
\hline Northeast & $429(18.7)$ & $429(18.7)$ & $541(17.7)$ & $541(17.7)$ & 0.3159 \\
\hline Midwest & $538(23.5)$ & $538(23.5)$ & $728(23.8)$ & $728(23.8)$ & 0.8120 \\
\hline South & $904(39.4)$ & $904(39.4)$ & $1251(40.8)$ & $1251(40.8)$ & 0.3102 \\
\hline West & $259(11.3)$ & $259(11.3)$ & $346(11.3)$ & $346(11.3)$ & 0.9896 \\
\hline Unknown & $162(7.1)$ & $162(7.1)$ & $199(6.5)$ & $199(6.5)$ & 0.4059 \\
\hline \multicolumn{6}{|l|}{ Type of healthcare plan } \\
\hline $\mathrm{PPO}$ & $1172(51.1)$ & $1172(51.1)$ & $1563(51.0)$ & $1563(51.0)$ & 0.9196 \\
\hline POS & $445(19.4)$ & $445(19.4)$ & $667(21.8)$ & $667(21.8)$ & $0.0362^{*}$ \\
\hline $\begin{array}{l}\text { Indemnity plan (i.e., fee- } \\
\text { for-service) }\end{array}$ & $312(13.6)$ & $312(13.6)$ & $321(10.5)$ & $321(10.5)$ & $0.0004^{*}$ \\
\hline $\mathrm{HMO}$ & $217(9.5)$ & $217(9.5)$ & $305(10.0)$ & $305(10.0)$ & 0.5550 \\
\hline Other $^{c}$ & $146(6.4)$ & $146(6.4)$ & $209(6.8)$ & $209(6.8)$ & 0.5134 \\
\hline
\end{tabular}

Values in table are presented as the mean \pm standard deviation with the median in square brackets or as a number with the percentage in parenthesis, as appropriate

$H M O$ Health Maintenance Organzation, HS Hidradenitis suppurativa, POS point of service plan, PPO preferred provider organization, organization

*Significant at $p<0.05$

a Because patients with milder and more severe HS were matched exactly 1:1 with HS-free control, only a comparison between patients with milder HS and patients with more severe HS was possible at baseline

b Age at the index date

c Other types of healthcare plans included exclusive provider organization, pharmacy network, independent practice association healthcare plans, locked in healthcare plans, and unknown plans

\section{Comorbidities Reported to be Associated with HS}

Comorbidities reported in the literature to be associated with HS were more frequently observed in the HS cohorts (mild and severe) than in the HSfree cohorts. All findings presented are significant at $p<0.0001$. The largest differences were observed for follicular occlusion diseases (HS-mild vs. matched HS-free controls: 27.4 vs. $6.4 \%$; HSsevere vs. matched HS-free controls: 44.8 vs. 


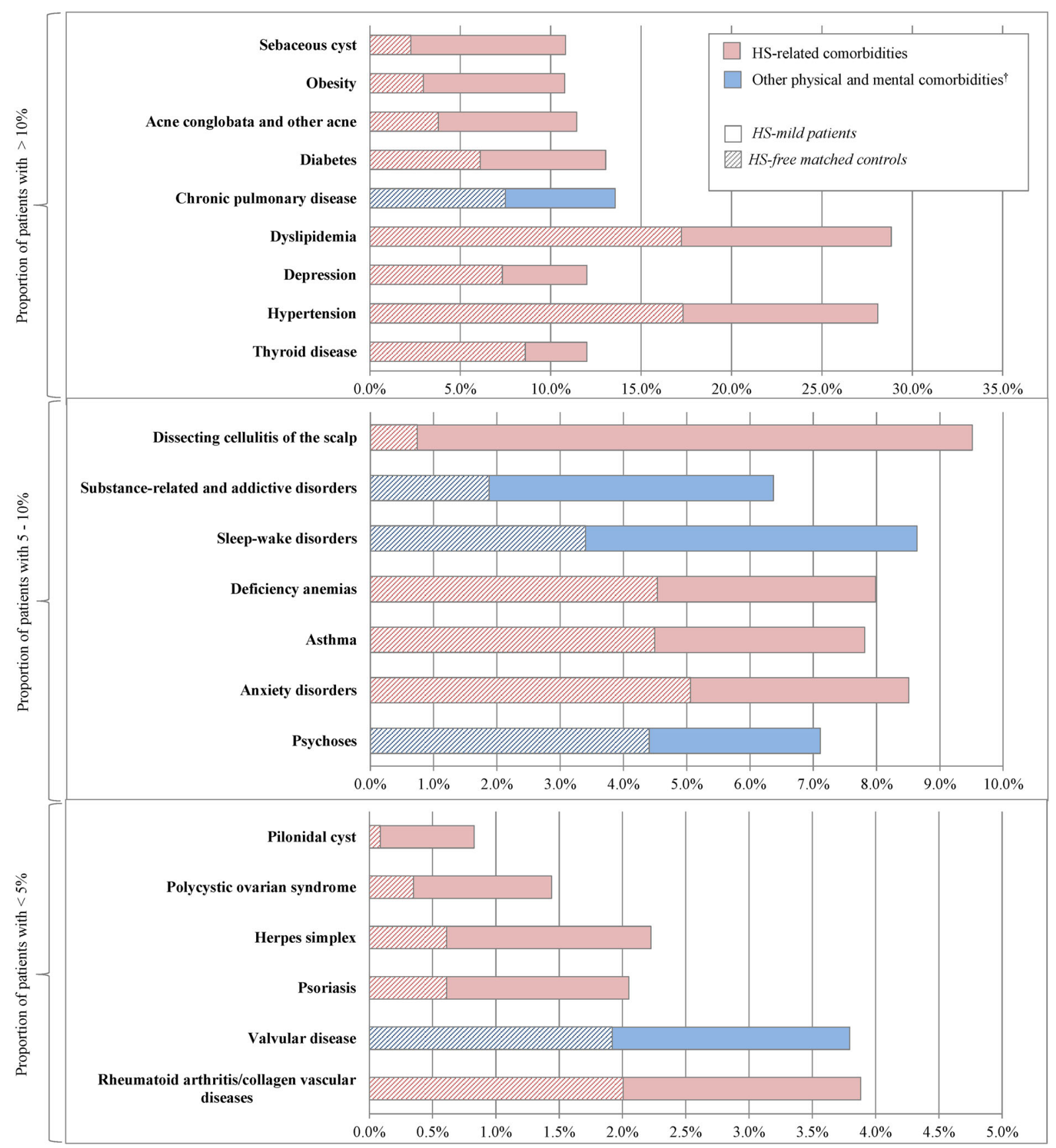

Fig. 2 Comparison of comorbid conditions between HSmild cohort and matched HS-free controls. All plotted effects are significant after Bonferroni correction to an overall level of 0.05 . Dagger indicates that other physical

$5.4 \%)$. Notably, when compared to their respective HS-free cohorts, pilonidal cyst was eightfold more prevalent in the HS-mild cohort and 35-fold and mental comorbidities were taken from Elixhauser et al. and the Diagnostic and Statistical Manual of Mental Disorders, 5th edition (DSM-5) [27-29]

more prevalent in the HS-severe cohort (HS-mild: 0.8 vs. $0.1 \%$; HS-severe: 3.5 vs. $0.1 \%$ ) (Figs. 2, 3; Electronic Supplementary Material Tables S1 and 


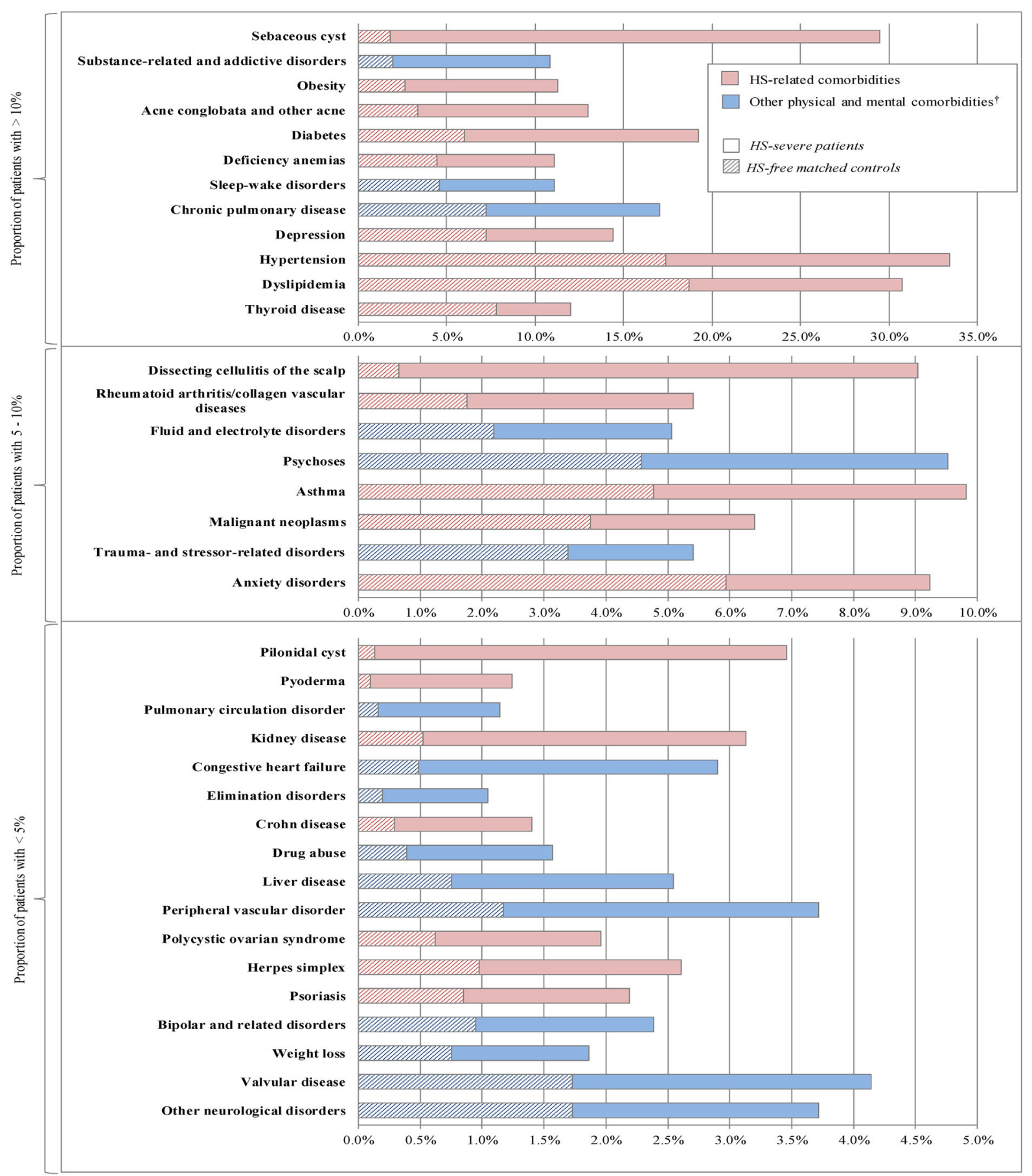

Fig. 3 Comparison of comorbid conditions between HSsevere cohort and matched HS-free controls. All plotted effects are significant after Bonferroni correction to an

S2). Also compared to their respective HS-free cohorts, dissecting cellulitis of the scalp (HS-mild: 9.5 vs. $0.7 \%$; HS-severe: 9.0 vs. $0.7 \%$ ) and overall level of 0.05 . Dagger indicates that other physical and mental comorbidities were taken from Elixhauser et al. and the DSM-5 [27-29]

sebaceous cyst (HS-mild: 10.8 vs. 2.3\%; HS-severe: 29.5 vs. $1.8 \%$ ) were also found to be more than fivefold prevalent in the HS-mild cohort and 
tenfold more prevalent in the HS-serve cohort. Additionally, acne conglobata and other acne (HS-mild: 11.4 vs. $3.8 \%$; HS-severe: 13.0 vs. $3.4 \%$ ) were over threefold more prevalent in both HS cohorts than in their respective HS-free controls.

Compared to their respective HS-free cohorts, inflammatory conditions, notably, rheumatoid arthritis/collagen vascular diseases (HS-mild: 3.9 vs. $2.0 \%$; HS-severe: 5.4 vs. $1.8 \%$ ) and psoriasis (HS-mild: 2.1 vs. $0.6 \%$; HS-severe: 2.2 vs. $0.8 \%$ ) were found to be almost threefold more prevalent in both HS cohorts. Additionally, in the HS-severe cohort, pyoderma (1.2 vs. $0.1 \%$ ) and Crohn's disease (HS-severe: 1.4 vs. $0.3 \%$ ) were found to be 12 -fold and fivefold, respectively more prevalent than in the HS-free cohort.

Endocrine/metabolic and hormone-related conditions, particularly obesity (HS-mild vs. matched HS-free controls: 10.8 vs. $3.0 \%$; HSsevere vs. matched HS-free controls: 11.3 vs. $2.6 \%$ ), diabetes (HS-mild: 13.0 vs. 6.1\%; HSsevere: 19.2 vs. $6.0 \%$ ), polycystic ovary syndrome (PCOS; HS-mild: 1.4 vs. 0.3\%; HS-severe: 2.0 vs. $0.6 \%$ ), hypertension (HS-mild: 28.1 vs. 17.3\%; HS-severe: 33.4 vs. $17.4 \%$ ), dyslipidemia (HS-mild: 28.8 vs. 17.2\%; HS-severe: 30.7 vs. $18.7 \%$ ), and thyroid disease (HS-mild: 12.0 vs. $8.6 \%$; HS-severe: 12.0 vs. $7.8 \%$ ) were all found to be more prevalent in the HS cohorts than in the HS-free cohorts.

Among other comorbidities reported in the literature to be associated with HS, herpes simplex (HS-mild vs. HS-free controls: 2.2 vs. $0.6 \%$; HS-severe vs. HS-free controls: 2.6 vs. $1.0 \%$ ), asthma (HS-mild: 7.8 vs. $4.5 \%$; HS-severe: 9.8 vs. $4.8 \%$ ), deficiency anemias (HS-mild: 8.0 vs. $4.5 \%$; HS-severe: 11.1 vs. $4.4 \%$ ), depression (HSmild: 12.0 vs. $7.3 \%$; HS-severe: 14.4 vs. $7.2 \%$ ), and anxiety disorders (HS-mild: 8.5 vs. $5.1 \%$; HS-severe: 9.2 vs. $5.9 \%$ ) were also found to be more prevalent in the HS cohorts than in the HS-free cohorts. Lastly, among patients with more severe forms of HS, kidney disease (HSsevere: 3.1 vs. $0.5 \%$ ) and non-cutaneous malignant neoplasms (HS-severe: 4.6 vs. $2.1 \%$ ) were also found to be significantly more prevalent than in the HS-free cohort.

\section{Other Comorbid Conditions}

Regarding other comorbidities, compared to the HS-free cohorts, physical and mental comorbidities [27, 28], chronic pulmonary disease (HS-mild: 13.6 vs. 7.5\%; HS-severe: 17.0 vs. $7.2 \%$ ), and valvular disease (HS-mild: 3.8 vs. $1.9 \%$; HS-severe: 4.1 vs. $1.7 \%$ ) were found to be more prevalent in the HS cohorts. Psychoses (HS-mild: 7.1 vs. $4.4 \%$; HS-severe: 9.5 vs. $4.6 \%$ ), substance-related and addictive disorders (HSmild: 6.4 vs. $1.9 \%$; HS-severe: 10.8 vs. $2.0 \%$ ), and sleep-wake disorders (HS-mild: 8.6 vs. $3.4 \%$; HS-severe: 11.1 vs. $4.6 \%$ ) were also found to be more than 1.5-fold more prevalent in the HS cohorts than in the HS-free cohorts.

In addition, among patients with more severe forms of HS, fluid and electrolyte disorders, pulmonary circulation disorder, congestive heart failure, drug abuse, liver disease, peripheral vascular disorder, weight loss, and other neurological disorders were found to be at least twofold more prevalent than in the HS-free cohort.

\section{Patients with Milder Versus More Severe Forms of HS}

Patients with more severe forms of HS were associated with an incremental burden compared to patients with milder forms of HS (Fig. 4). Notably, among comorbidities typically reported in the literature to be associated with HS, pilonidal cyst was over fourfold more prevalent in patients with more severe forms of HS than in patients with milder forms of HS (3.5 vs. $0.8 \%)$. Sebaceous cyst (29.5 vs. $10.8 \%)$ and kidney disease (3.1 vs. $1.5 \%$ ) were also over twofold more prevalent in patients with more severe forms of HS. Hypertension (33.4 vs. $28.1 \%)$, diabetes (19.2 vs. $13.0 \%)$, and deficiency anemias (11.1 vs. $8.0 \%$ ) were also found to be more prevalent in patients with more severe forms of HS. Among other comorbidities not typically reported in the literature to be associated with HS, congestive heart failure (2.9 vs. $1.0 \%$ ) and substance-related and addictive disorders (10.8 vs. $6.4 \%)$ were, 2.9 -fold and 1.7fold more prevalent in patients with more 


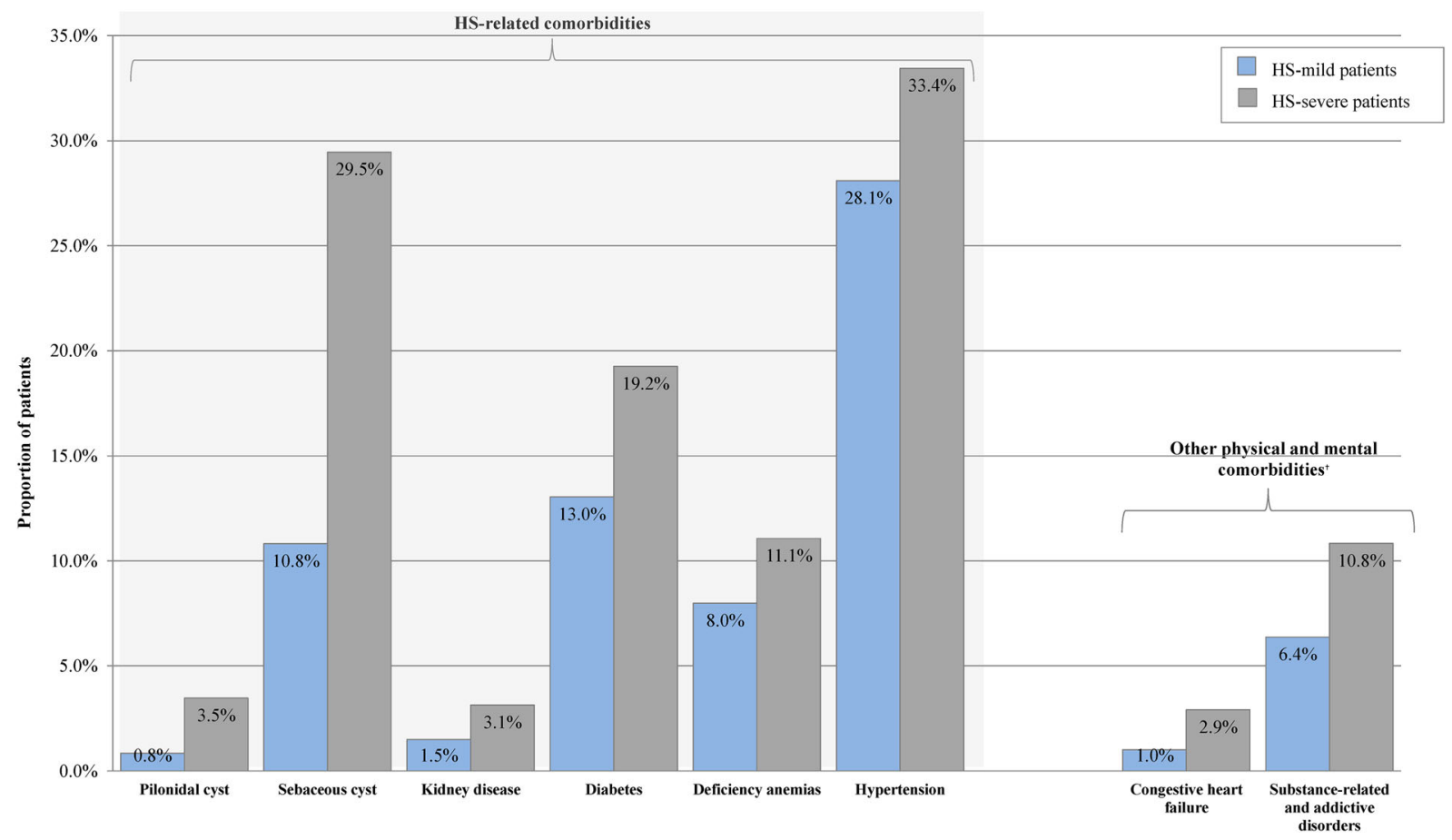

Fig. 4 Comparison of comorbid characteristics between HS-severe and HS-mild cohorts. All plotted effects are significant after Bonferroni correction to an overall level of

severe forms of HS than in patients with milder forms of HS.

\section{DISCUSSION}

Although prior studies have shown that HS is accompanied by a myriad of comorbid conditions $[7,30]$, the generalizability of the results from prior studies is generally limited by the small sample sizes and the limited number of pre-selected comorbidities. In addition, despite the fact that the comorbidity burden of HS is likely to vary with the severity of the disease, the relationship between HS severity and the comorbidity burden has not been yet characterized from an epidemiological perspective. In this study, we assessed the comorbidity burden of HS, stratified by indicators of disease severity, in a large sample of US patients, assessing more than 60 comorbid conditions. Our results show that the comorbidity burden among HS patients
0.05. Dagger indicates that other physical and mental comorbidities were taken from Elixhauser et al. and the DSM-5 [27-29]

is substantial and becomes increasingly more intense in patients with severe forms.

Several physical and mental comorbidities were found to be more prevalent in both patients with milder and those with more severe forms of HS compared to their matched controls, in agreement with findings reported in the literature $[5,7,8,13-18,31-33]$. These comorbidities included follicular occlusion conditions (acne conglobata, dissecting cellulitis of the scalp, and pilonidal cyst), endocrine, metabolic, and hormone-related disorders (diabetes, obesity, dyslipidemia, PCOS, and thyroid disease), cardiovascular disorders, such as hypertension, inflammatory conditions (psoriasis and rheumatoid arthritis/collagen vascular disorder), psychiatric disorders (depression and anxiety), as well as other diseases, such as deficiency anemia, asthma, and herpes simplex.

Although the present study included a general definition of thyroid disease (i.e., comprising both hyperthyroidism and hypothyroidism), compared to the matched controls hyperthyroidism 
(HS-severe: 1.4 vs. $0.5 \%$ ) and hypothyroidism (HS-severe: 9.1 vs. $6.1 \%$ ) were each found to be more prevalent in patients with more severe forms of HS. The association observed between hyperthyroidism and HS is in agreement with published results, but the association observed between hypothyroidism and HS contrasts what has been previously reported [34]. However, drawing direct parallels between the results of this study and those of previous studies is challenging due to differences in study designs, data sources, and sample populations. In addition, there are conflicting reports regarding the associations between HS and Hashimoto's thyroiditis and hypothyroidism [35-37]. In the present study, there was no significant association observed between Hashimoto's thyroiditis and HS.

Other comorbidities, such as valvular disease, chronic pulmonary disease, sleep/wake disorders, substance-related/addictive disorders, and psychoses, were also observed to be more prevalent in both patients with milder and more severe forms of HS than in their matched controls. Additional physical and mental comorbidities were found to be more prevalent among patients with more severe forms of HS than in their matched controls. Some of these comorbidities, such as kidney disease, Crohn's disease, malignant neoplasms, pyoderma gangrenosum, bipolar disorder, and drug abuse have been previously reported $[7,11,13,18,30,32,38-40]$, while others, such as fluid and electrolyte disorders, other neurological disorders, congestive heart failure, pulmonary circulation disorder, peripheral vascular disorder, liver disease, weight loss, elimination disorders, and trauma/stress-related disorders, have not.

The higher prevalence of comorbidities, including follicular occlusion conditions, metabolic syndrome, and inflammatory conditions in HS patients, could potentially be explained by the common inflammatory mechanisms and immune dysregulation typically implicated in autoimmune disorders [41]. Notably, HS patients have been shown to have elevated levels of systemic inflammation that surpass those of psoriasis [42]. It has also been hypothesized that the deep-seated chronic inflammation observed in HS may be associated with atrophy of the sebaceous glands and follicular hyperkeratinization and destruction [41], in accordance with the recent definition of HS as an autoinflammatory keratinization disease [43]. To that extent, it is likely that the more severe forms of HS may relate to greater increases in systemic inflammation and be associated with a greater number, and proportion, of comorbidities observed in HS. Indeed, the results of this study suggest that the comorbidity burden of HS increases in more severe forms of the disease and that this burden may be cumulative in advanced stages. Several comorbidities were found to be more prevalent in patients with more severe forms of HS, such as pilonidal cyst, sebaceous cyst, deficiency anemia, kidney disease, diabetes, and hypertension, which have previously been associated with HS, as well as other comorbidities, such as congestive heart failure and substance-related/ addictive disorders.

\section{Limitations}

Although the results of the present study indicate an increased comorbidity burden associated with HS and more so with more severe forms of HS, it must be noted that some patients with the milder forms of HS may have been untreated, which may indicate a more conservative estimate of the comorbidity burden associated with HS.

Since claims data do not provide information on disease severity, our analytic approach was limited in terms of identifying patients with milder and more severe HS. Patients with these forms were identified using an empirical algorithm based on the types of treatments received; potential misclassification of patients as having milder or more severe forms of HS may have yielded an overestimation of the comorbidity burden for patients with milder forms of HS or an underestimation of the comorbidity burden for patients with more severe forms of HS. The rate for some of the study comorbid conditions may have been underestimated, for example, in cases of patients whose comorbid conditions was managed at home and for comorbid conditions that did not require medical attention. Although the database utilized in this study contained approximately 14 million privately insured 
beneficiaries, across 60 large US corporations, it is limited to privately insured employees and their dependents. Access to care may be different for patients who are not privately insured, and it is unclear if their comorbidity burden might differ.

\section{CONCLUSIONS}

Overall, the results from this study are in line with those from prior studies showing a substantial comorbidity burden among patients with HS. They also suggest that the comorbidity burden increases with disease severity. As many of the comorbid conditions observed in patients with HS belong to a wide range of clinical categories, our results suggest that the management of HS and its numerous comorbidities might require a multidisciplinary approach involving the coordinated effort of a team of specialists. In this context, a better understanding of which comorbidities are most frequently encountered at different stages of HS is particularly important to help physicians plan and optimize treatment strategies [4].

\section{ACKNOWLEDGEMENTS}

Funding. Sponsorship for this study and article processing charges were funded by AbbVie. AbbVie participated in the study design, study conduct, interpretation of data, and review. All authors had full access to all of the data in this study and take complete responsibility for the integrity of the data and accuracy of the data analysis.

Medical Writing, Editorial, and Other Assistance. Medical writing assistance was provided by Cinzia Metallo, $\mathrm{PhD}$, an employee of Analysis Group, Inc. Support for this assistance was funded by AbbVie.

Authorship. All named authors meet the International Committee of Medical Journal Editors (ICMJE) criteria for authorship for this article, take responsibility for the integrity of the works as a whole, and have given their approval for this version to be published.

Disclosures. Alexandra B. Kimball received honoraria as a consultant and grants as an investigator from Janssen, AbbVie, UCB, and Novartis, and has received fellowship funding from Janssen and AbbVie. Arijit Ganguli is an employee of AbbVie and may own stock/stock options. Rakesh Singh is an employee of AbbVie and may own stock/stock options. Murali Sundaram is now an employee of Johnson \& Johnson, New Brunswick, NJ. Genevieve Gauthier was an employee of Analysis Group, Inc at the time the study was conducted. Genevieve Gauthier is now an employee of StatLog Inc., Montreal, Canada. Annie Guérin is an employee of Analysis Group Inc, which has received consultancy fees from AbbVie. Irina Piveneva is an employee of Analysis Group Inc, which has received consultancy fees from AbbVie. Genevieve Gauthier is a former employee of Analysis Group Inc., which has received consultancy fees from AbbVie.

Compliance with Ethics Guidelines. This was a retrospective analysis of anonymized data; no institutional board review was required.

Open Access. This article is distributed under the terms of the Creative Commons AttributionNonCommercial 4.0 International License (http:// creativecommons.org/licenses/by-nc/4.0/), which permits any noncommercial use, distribution, and reproduction in any medium, provided you give appropriate credit to the original author(s) and the source, provide a link to the Creative Commons license, and indicate if changes were made.

\section{REFERENCES}

1. Jemec GB. Clinical practice. Hidradenitis suppurativa. N Engl J Med. 2012;366(2):158-64.

2. Saunte DML, Jemec GBE. Hidradenitis suppurativa: advances in diagnosis and treatment. JAMA. 2017;318(20):2019-32.

3. Jemec GB, Heidenheim M, Nielsen NH. Hidradenitis suppurativa-characteristics and consequences. Clin Exp Dermatol. 1996;21(6):419-23. 
4. Menter A. Recognizing and managing comorbidities and complications in hidradenitis suppurativa. Semin Cutan Med Surg. 2014;33[3 Suppl]:S54-6.

5. Scheinfeld NS. A case of dissecting cellulitis and a review of the literature. Dermatol Online J. 2003;9:87-93.

6. Chicarilli ZN. Follicular occlusion triad: hidradenitis suppurativa, acne conglobata, and dissecting cellulitis of the scalp. Ann Plast Surg. 1987;18(3):230-7.

7. Fimmel S, Zouboulis C. Comorbidities of hidradenitis suppurativa (acne inversa). Dermatoendocrinology. 2010;2(1):9-16.

8. Ingelfinger JR, Seifter JL. Integration of acid-base and electrolyte disorders. N Engl J Med. 2014;371(19): 1821-31.

9. Patel M, Cohen JM, Wright NA, Merola JF, Qureshi AA, Vleugels RA. Epidemiology of concomitant psoriasis and hidradenitis suppurativa (HS): experience of a tertiary medical center. J Am Acad Dermatol. 2015;73(4):701-2.

10. Jansen I, Altmeyer P, Piewig G. Acne inversa (alias hidradenitis suppurativa). J Eur Acad Dermatol Venereol. 2001;15(6):532-40.

11. Scheinfeld N. Diseases associated with hidradenitis suppurativa: part 2 of a series on hidradenitis. Dermatol Online J. 2013;19(6):18558.

12. van der Zee $H H$, van der Woude CJ, Florencia EF, Prens EP. Hidradenitis suppurativa and inflammatory bowel disease: are they associated? Results of a pilot study. Br J Dermatol. 2010;162(1):195-7.

13. Kohorst JJ, Kimball AB, Davis MD. Systemic associations of hidradenitis suppurativa. J Am Acad Dermatol. 2015;73(5 Suppl 1):S27-35.

14. Danby FW, Margesson LJ. Hidradenitis suppurativa. Dermatol Clin. 2010;28(4):779-93.

15. Shah N. Hidradenitis suppurativa: a treatment challenge. Am Fam Physician. 2005;72(8):1547-52.

16. Shlyankevich J, Chen AJ, Kim GE, Kimball AB. Hidradenitis suppurativa is a systemic disease with substantial comorbidity burden: a chart-verified case-control analysis. J Am Acad Dermatol. 2014;71(6):1144-50.

17. Hsiao JL, Antaya RJ, Berger T, Maurer T, Shinkai K, Leslie KS. Hidradenitis suppurativa and concomitant pyoderma gangrenosum: a case series and literature review. Arch Dermatol. 2010;146(11): 1265-70.
18. Wollina U, Tilp M, Meseg A, Schonlebe J, Heinig B, Nowak A. Management of severe anogenital acne inversa (hidradenitis suppurativa). Dermatol Surg. 2012;38(1):110-7.

19. Mebazaa A, Ben Hadid R, Cheikh Rouhou R, Trojjet S, El Euch D, Mokni M, et al. Hidradenitis suppurativa: a disease with male predominance in Tunisia. Acta Dermatovenerol Alp Pannonica Adriat. 2009;18(4):165-72.

20. Melnik BC, John SM, Chen W, Plewig G. T helper 17 cell/regulatory T-cell imbalance in hidradenitis suppurativa/acne inversa: the link to hair follicle dissection, obesity, smoking and autoimmune comorbidities. Br J Dermatol. 2018;179:260-72.

21. De Vita V, Melnik BC. Activated mTORC1 signaling: the common driving force of type 2 diabetes and hidradenitis suppurativa. J Am Acad Dermatol. 2018;78(5):e121.

22. De Vita V, Hansen PR. Interleukin-32 may contribute to increased risk of cardiovascular disease in patients with hidradenitis suppurativa. Atherosclerosis. $2017 ; 267: 175$.

23. De Vita V, Ruocco E. The concept of immunocompromised district might explain the carcinogenic progression in hidradenitis suppurativa. Acta Oncol. 2017;56(10):1330-1.

24. De Vita V, Ruocco E. Letter to the Editor referring to Hessam et al.-squamous cell carcinoma arising in hidradenitis suppurativa: subclinical lymphedema may have favored the tumor onset. J Dtsch Dermatol Ges. 2017;15(5):580-2.

25. De Vita V, Ruocco E. Mucinous adenocarcinoma in association with hidradenitis suppurativa: a new example of isoscartopic response. Clin Exp Dermatol. $2018 ; 43(4): 472-3$.

26. Bettoli V, Naldi L, Cazzaniga S, Zauli S, Atzori L, Borghi A, et al. Overweight, diabetes and disease duration influence clinical severity in hidradenitis suppurativa-acne inversa: evidence from the national Italian registry. $\mathrm{Br} \mathrm{J}$ Dermatol. 2016;174(1):195-7.

27. Quan H, Sundararajan V, Halfon P, Fong A, Burnand $\mathrm{B}$, Luthi JC, et al. Coding algorithms and for defining comorbidities data in ICD-9-CM administrative. Med Care. 2005;43(11):1130-9.

28. American Psychiatric Association. Diagnostic and statistical manual of mental disorders, fifth edition. DSM-5. Arlington: American Psychiatric Publishing; 2013. 
29. Elixhauser A, Steiner C, Harris DR, Coffey RM. Comorbidity measures for use with administrative data. Med Care. 1998;36(1):8-27

30. Dufour DN, Emtestam L, Jemec GB. Hidradenitis suppurativa: a common and burdensome, yet under-recognised, inflammatory skin disease. Postgrad Med J. 2014;90(1062):216-21 (quiz 20).

31. Shavit E, Dreiher J, Freud T, Halevy S, Vinker S, Cohen AD. Psychiatric comorbidities in 3207 patients with hidradenitis suppurativa. J Eur Acad Dermatol Venereol. 2015;29(2):371-6.

32. Miller IM, McAndrew RJ, Hamzavi I. Prevalence, risk factors, and comorbidities of hidradenitis suppurativa. Dermatol Clin. 2016;34(1):7-16.

33. Wolkenstein P, Loundou A, Barrau K, Auquier P, Revuz J. Quality of life impairment in hidradenitis suppurativa: a study of 61 cases. J Am Acad Dermatol. 2007;56(4):621-3.

34. Miller IM, Vinding G, Sorensen HA, Rytgaard H, Mogensen UB, Ellervik C, et al. Thyroid function in hidradenitis suppurativa: a population-based crosssectional study from Denmark. Clin Exp Dermatol. 2018. https://doi.org/10.1111/ced.13606.

35. Gonzalez-Lopez MA, Blanco R, Mata C, Lopez-Escobar M, Lacalle M, Consuegra G, et al. Coexistence of hidradenitis suppurativa with autoimmune thyroiditis: report of three cases. Dermatology. 2016;232(2):162-4.

36. Gonzalez-Lopez MA, Hernandez JL, Vilanova I, Mata C, Lopez-Escobar M, Gonzalez-Vela MC, et al. Thyroid autoimmunity in patients with hidradenitis suppurativa: a case-control study. Clin Exp Dermatol. 2017;42(6):642-4.
37. De Vita V, McGonagle D. The immunological disease continuum of inflammation against self as an explanation for the lack of association between hidradenitis suppurativa and autoimmune thyroid disease. Clin Exp Dermatol. 2018;43(5):601-3.

38. Gill L, Williams M, Hamzavi I. Update on hidradenitis suppurativa: connecting the tracts. F1000Prime Rep. 2014;6:112.

39. Huilaja L, Tiri H, Jokelainen J, Timonen M, Tasanen K. Patients with hidradenitis suppurativa have a high psychiatric disease burden: a finnish nationwide registry study. J Invest Dermatol. 2018;138(1):46-51.

40. Garg A, Papagermanos V, Midura M, Strunk A, Merson J. Opioid, alcohol, and cannabis misuse among patients with hidradenitis suppurativa: a population-based analysis in the United States. J Am Acad Dermatol. 2018;79(3):495-500.e1.

41. Egeberg A, Gislason GH, Hansen PR. Risk of major adverse cardiovascular events and all-cause mortality in patients with hidradenitis suppurativa. JAMA Dermatol. 2016;152(4):429-34.

42. Riis PT, Soeby K, Saunte DM, Jemec GB. Patients with hidradenitis suppurativa carry a higher systemic inflammatory load than other dermatological patients. Arch Dermatol Res. 2015;307(10):885-9.

43. De Vita V, McGonagle D. Hidradenitis suppurativa as an autoinflammatory keratinization disease. J Allergy Clin Immunol. 2018;141(5):1953. 